\title{
Palindromic continued fractions
}

\author{
Boris Adamczewski (Lyon) \& Yann Bugeaud * (Strasbourg)
}

\section{Introduction}

It is widely believed that the continued fraction expansion of every irrational algebraic number $\alpha$ is either eventually periodic (and this is the case if, and only if, $\alpha$ is a quadratic irrationality) or contains arbitrarily large partial quotients, but we seem to be very far away from a proof (or a disproof). A preliminary step consists in providing explicit examples of transcendental continued fractions. The first result of this type is due to Liouville [11], who constructed real numbers whose sequence of partial quotients grows very fast, too fast for being algebraic. Subsequently, various authors used deeper transcendence criteria from Diophantine approximation to construct other classes of transcendental continued fractions. Of particular interest is the work of Maillet [12] (see also Section 34 of Perron [13]), who was the first to give examples of transcendental continued fractions with bounded partial quotients. Further examples have been provided by Baker [4, 5], Davison [6], Queffélec [14], Allouche et al. [3] and Adamczewski and Bugeaud [1], among others.

A common feature of the above quoted results is that they apply to real numbers whose continued fraction expansion is 'quasi-periodic', in the sense that it contains arbitrarily long blocks of partial quotients which occur precociously at least twice. In the present work, we investigate real numbers whose sequence of partial quotients enjoys another combinatorial property, namely is 'quasi-symmetrical', in the sense that it begins in arbitrarily large 'quasi-palindromes'. We provide three new transendence criteria, that apply to a broad class of continued fraction expansions, including expansions with unbounded partial quotients. As in [1], their proofs heavily depend on the Schmidt Subspace Theorem. These criteria are stated in Section 2 and proved in Sections 6 and 7 . Our method allows us to improve upon an earlier result of A. Baker [4] on the transcendence of continued fractions whose sequence of partial quotients is composed of long strings on 1 and long strings of 2, alternatively. See Section 3 for a precise statement and Section 8 for its proof. In Section 4, we provide an application of one of our transcendence criteria to the explicit construction of transcendental numbers with sharp properties of approximation by rational numbers. All our auxiliary statements are gathered in Section 5.

\footnotetext{
* Supported by the Austrian Science Fundation FWF, grant M822-N12.
} 


\section{Main results}

Throughout the present work, $\mathcal{A}$ denotes a given set, not necessarily finite. We identify any sequence $\mathbf{a}=\left(a_{n}\right)_{n \geq 1}$ of elements from $\mathcal{A}$ with the infinite word $a_{1} a_{2} \ldots a_{n} \ldots$ Recall that a finite word $a_{1} a_{2} \ldots a_{n}$ on $\mathcal{A}$ is called a palindrome if $a_{j}=a_{n+1-j}$ for $j=1, \ldots, n$.

Our first transcendence criterion can be stated as follows.

Theorem 1. Let $\mathbf{a}=\left(a_{n}\right)_{n \geq 1}$ be a sequence of positive integers. If the word a begins in arbitrarily long palindromes, then the real number $\alpha:=\left[0 ; a_{1}, a_{2}, \ldots, a_{n}, \ldots\right]$ is either quadratic irrational or transcendental.

We point out that there is no assumption on the growth of the sequence $\left(a_{n}\right)_{n \geq 1}$ in Theorem 1, unlike in Theorems 2 and 3 below.

As shown in [2], given two distinct positive integers $a$ and $b$, Theorem 1 easily implies the transcendence of the real number $\left[0 ; a_{1}, a_{2}, \ldots \ldots.\right]$, whose sequence of partial quotients is the Thue-Morse sequence on the alphabet $\{a, b\}$, that is, with $a_{n}=a\left(\right.$ resp. $\left.a_{n}=b\right)$ if the sum of binary digits of $n$ is odd (resp. even). This result is originally due to M. Queffélec [14].

Before stating our next theorems, we need to introduce some more notation. The length of a finite word $W$ on the alphabet $\mathcal{A}$, that is, the number of letters composing $W$, is denoted by $|W|$. The mirror image of $W:=a_{1} \ldots a_{n}$ is the word $\bar{W}:=a_{n} \ldots a_{1}$. In particular, $W$ is a palindrome if, and only if, $W=\bar{W}$. A palindrome is thus a finite word invariant under mirror symmetry. In order to relax this property of symmetry, we introduce the notion of quasi-palindrome. Let $U$ and $V$ be two finite words; then, the word $U V \bar{U}$ is called a quasi-palindrome of order $w$, where $w=|V| /|U|$. Clearly, the larger $w$ is, the weaker is the property of symmetry. In our next transcendence criterion, we replace the occurrences of aritrarily large palindromes by the ones of arbitrarily large quasi-palindromes of bounded order. However, this weakening of our assumption has a cost, namely, an extra assumption on the growth of the partial quotients is then needed. Fortunately, the latter assumption is not very restrictive. In particular, it is always satisfied by real numbers with bounded partial quotients.

Let $\mathbf{a}=\left(a_{n}\right)_{n \geq 1}$ be a sequence of elements from $\mathcal{A}$. Let $w$ be a rational number with $w>1$. We say that a satisfies Condition $(*)_{w}$ if $\mathbf{a}$ is not eventually periodic and if there exist two sequences of finite words $\left(U_{n}\right)_{n \geq 1}$ and $\left(V_{n}\right)_{n \geq 1}$ such that:

(i) For any $n \geq 1$, the word $U_{n} V_{n} \bar{U}_{n}$ is a prefix of the word a;

(ii) The sequence $\left(\left|V_{n}\right| /\left|U_{n}\right|\right)_{n \geq 1}$ is bounded from above by $w$;

(iii) The sequence $\left(\left|U_{n}\right|\right)_{n \geq 1}$ is increasing.

We complement Theorem 1 in the following way.

Theorem 2. Let $\mathbf{a}=\left(a_{n}\right)_{n \geq 1}$ be a sequence of positive integers. Let $\left(p_{n} / q_{n}\right)_{n \geq 1}$ denote the sequence of convergents to the real number

$$
\alpha:=\left[0 ; a_{1}, a_{2}, \ldots, a_{n}, \ldots\right] .
$$


Assume that the sequence $\left(q_{\ell}^{1 / \ell}\right)_{\ell \geq 1}$ is bounded, which is in particular the case when the sequence $\mathbf{a}$ is bounded. If there exists a positive rational number $w$ such that a satisfies Condition $(*)_{w}$, then $\alpha$ is transcendental.

In the statements of Theorems 1 and 2 the palindromes or the quasi-palindromes must appear at the very beginning of the continued fraction under consideration. Fortunately, the ideas used in their proofs allow us to deal also with the more general situation where arbitrarily long quasi-palindromes occur not too far from the beginning.

Let $w$ and $w^{\prime}$ be positive rational numbers. We say that a satisfies Condition $(*)_{w, w^{\prime}}$ if $\mathbf{a}$ is not eventually periodic and if there exist three sequences of finite words $\left(U_{n}\right)_{n \geq 1}$, $\left(V_{n}\right)_{n \geq 1}$ and $\left(W_{n}\right)_{n \geq 1}$ such that:

(i) For any $n \geq 1$, the word $W_{n} U_{n} V_{n} \bar{U}_{n}$ is a prefix of the word a;

(ii) The sequence $\left(\left|V_{n}\right| /\left|U_{n}\right|\right)_{n \geq 1}$ is bounded from above by $w$;

(iii) The sequence $\left(\left|U_{n}\right| /\left|W_{n}\right|\right)_{n \geq 1}$ is bounded from below by $w^{\prime}$;

(iv) The sequence $\left(\left|U_{n}\right|\right)_{n \geq 1}$ is increasing.

We are now ready to complement Theorems 2 and 3 as follows.

Theorem 3. Let $\mathbf{a}=\left(a_{n}\right)_{n \geq 1}$ be a sequence of positive integers. Let $\left(p_{n} / q_{n}\right)_{n \geq 1}$ denote the sequence of convergents to the real number

$$
\alpha:=\left[0 ; a_{1}, a_{2}, \ldots, a_{n}, \ldots\right]
$$

Assume that the sequence $\left(q_{\ell}^{1 / \ell}\right)_{\ell \geq 1}$ is bounded and set $M=\limsup _{\ell \rightarrow+\infty} q_{\ell}^{1 / \ell}$ and $m=$ $\lim \inf _{\ell \rightarrow+\infty} q_{\ell}^{1 / \ell}$. Let $w$ and $w^{\prime}$ be positive rational numbers with

$$
w^{\prime}>2 \frac{\log M}{\log m}-1
$$

If a satisfies Condition $(*)_{w, w^{\prime}}$, then $\alpha$ is transcendental.

We display an immediate consequence of Theorem 3.

Corollary 1. Let $\mathbf{a}=\left(a_{n}\right)_{n \geq 1}$ be a sequence of positive integers. Let $\left(p_{n} / q_{n}\right)_{n \geq 1}$ denote the sequence of convergents to the real number

$$
\alpha:=\left[0 ; a_{1}, a_{2}, \ldots, a_{n}, \ldots\right]
$$

Assume that the sequence $\left(q_{\ell}^{1 / \ell}\right)_{\ell \geq 1}$ converges. Let $w$ and $w^{\prime}$ be positive rational numbers with $w^{\prime}>1$. If a satisfies Condition $(*)_{w, w^{\prime}}$, then $\alpha$ is transcendental.

Theorems 1 to 3 provide the exact analogues of Theorems 1 and 2 from [1]. It would be desirable to replace the assumption $(2.1)$ by the weaker one $w^{\prime}>0$. The statements of Theorems 2 and 3 show that weakening the combinatorial assumption of Theorem 1 needs further assumptions on the size of the partial quotients. 


\section{On a theorem of A. Baker}

In this Section we focus on a particular family of continued fractions introduced by Baker [4]. Let $a$ and $b$ be distinct positive integers. Let $\boldsymbol{\Lambda}=\left(\lambda_{n}\right)_{n \geq 1}$ be a sequence of positive integers and set

$$
\alpha_{\Lambda}:=\left[0 ; \bar{a}^{\lambda_{1}}, \bar{b}^{\lambda_{2}}, \bar{a}^{\lambda_{3}}, \ldots, \bar{b}^{\lambda_{2 n}}, \bar{a}^{\lambda_{2 n+1}}, \ldots\right]
$$

where, for positive integers $m$ and $\lambda$, we use the notation $\bar{m}^{\lambda}$ to denote a string of $\lambda$ consecutive partial quotients equal to $m$. In his paper, Baker proved the transcendence of $\alpha_{\boldsymbol{\Lambda}}$ assuming that the sequence $\boldsymbol{\Lambda}$ increases sufficiently rapidly.

For convenience, we assume that $b>a$ (this makes no restriction, since the transcendence of a number does not depend on his first partial quotients), and we set

$$
\alpha_{a}=[a ; a, a, \ldots], \quad \alpha_{b}=[b ; b, b, \ldots],
$$

and

$$
\rho=\log \alpha_{b} / \log \alpha_{a}
$$

Observe that we have $\rho>1$.

Although stated in a weaker form, the following result can be derived from [4].

Theorem. (A. Baker). Let $\alpha_{\boldsymbol{\Lambda}}$ be as above. If the sequence $\boldsymbol{\Lambda}$ satisfies

$$
\liminf _{n \rightarrow \infty} \frac{\lambda_{n+1}}{\lambda_{n}}>\frac{1+\sqrt{8 \rho^{2}+1}}{2 \rho},
$$

then $\alpha_{\boldsymbol{\Lambda}}$ is transcendental.

Baker's proof rests on the generalisation, due to LeVeque [10], of Roth's Theorem to approximation by algebraic numbers from a given number field. Using our approach based on quasi-palindromes and on the Schmidt Subspace Theorem, we are able to slightly improve upon his result.

Theorem 4. Let $\alpha_{\boldsymbol{\Lambda}}$ be as above. If the sequence $\boldsymbol{\Lambda}$ satisfies

$$
\liminf _{n \rightarrow \infty} \frac{\lambda_{n+1}}{\lambda_{n}}>\sqrt{2}
$$

then $\alpha_{\boldsymbol{\Lambda}}$ is transcendental.

Theorem 4 improves upon Baker's result, since we have

$$
\sqrt{2}<\frac{1+\sqrt{8 \rho^{2}+1}}{2 \rho}<2,
$$

for any $\rho>1$. For instance, taking $a=1$ and $b=2$, Baker obtained the value $1.71 \ldots$ (cf. page 8 of [4]). Throughout the proof of Theorem 4 (postponed to Section 8), we will see how it is often possible to refine the assumption (2.1) of Theorem 3. 


\section{Transcendental numbers with prescribed order of approximation}

In Satz 6 of [7], Jarník used the continued fraction theory to prove the existence of real numbers with prescribed order of approximation by rational numbers. Let $\varphi: \mathbf{R}_{\geq 1} \rightarrow \mathbf{R}_{>0}$ be a positive function. We say that a real number $\alpha$ is 'approximable at order $\varphi$ ' if there exist infinitely many rational numbers $p / q$ with $q>0$ and $|\alpha-p / q|<\varphi(q)$. Jarník's result can then be stated as follows.

Theorem J. Let $\varphi: \mathbf{R}_{\geq 1} \rightarrow \mathbf{R}_{>0}$ be a non-increasing function such that $\varphi(x)=o\left(x^{-2}\right)$ as $x$ tends to infinity. Then, there are real numbers $\alpha$ which are approximable at order $\varphi$ but which are not approximable at any order $c \varphi$, with $0<c<1$.

In his proof, Jarník constructed inductively the sequence of partial quotients of $\alpha$. Actually, he showed that there are uncountably many real numbers $\alpha$ with the required property, thus, in particular, transcendental numbers. However, his construction did not provide any explicit example of such a transcendental $\alpha$.

In the present Section, we apply our Theorem 1 to get, under an extra assumption on the function $\varphi$, explicit examples of transcendental numbers satisfying the conclusion of Theorem J.

Theorem 5. Let $\varphi: \mathbf{R}_{>1} \rightarrow \mathbf{R}_{>0}$ be such that $x \mapsto x^{2} \varphi(x)$ is non-increasing and tends to 0 as $x$ tends to infinity. Then, we can construct explicit examples of transcendental numbers $\alpha$ which are approximable at order $\varphi$ but which are not approximable at any order $c \varphi$, with $0<c<1$.

Proof : Throughout the proof, for any real number $x$, we denote by $\lceil x\rceil$ the smallest integer greater than or equal to $x$. We will construct inductively the sequence $\left(b_{n}\right)_{n \geq 1}$ of partial quotients of a suitable real number $\alpha$. Denoting by $\left(p_{n} / q_{n}\right)_{n \geq 0}$ the sequence of convergents to $\alpha$, it follows from the continued fraction theory that, for any $n \geq 1$, we have

$$
\frac{1}{q_{n-1}^{2}\left(b_{n}+2\right)}<\left|\alpha-\frac{p_{n-1}}{q_{n-1}}\right|<\frac{1}{q_{n-1}^{2} b_{n}} .
$$

Recall that $q_{n} \geq(3 / 2)^{n}$ for any $n \geq 5$. For any $x \geq 1$, set $\Psi(x)=x^{2} \varphi(x)$. Let $n_{1} \geq 6$ be such that $\Psi\left((3 / 2)^{n}\right) \leq 10^{-1}$ for any $n \geq n_{1}-1$. Then, set $b_{1}=\ldots=b_{n_{1}-1}=1$ and $b_{n_{1}}=$ $\left\lceil 1 / \Psi\left(q_{n_{1}-1}\right)\right\rceil$. Observe that $b_{n_{1}} \geq 10$. Let $n_{2}>n_{1}$ be such that $\Psi\left((3 / 2)^{n}\right) \leq\left(10 b_{n_{1}}\right)^{-1}$ for any $n \geq n_{2}-1$. Then, set $b_{n_{1}+1}=\ldots=b_{n_{2}-1}=1$ and $b_{n_{2}}=\left\lceil 1 / \Psi\left(q_{n_{2}-1}\right)\right\rceil$. Observe that $b_{n_{2}} \geq 10 b_{n_{1}}$.

At this step, we have

$$
\alpha=\left[0 ; \overline{1}^{n_{1}-1}, b_{n_{1}}, \overline{1}^{n_{2}-n_{1}-1}, b_{n_{2}}, \ldots\right],
$$

where, as in the previous Section, we denote by $\overline{1}^{m}$ a sequence of $m$ consecutive partial quotients equal to 1 . Then, we complete by symmetry, in such a way that the continued fraction expansion of $\alpha$ begins with a palindrome:

$$
\alpha=\left[0 ; \overline{1}^{n_{1}-1}, b_{n_{1}}, \overline{1}^{n_{2}-n_{1}-1}, b_{n_{2}}, \overline{1}^{n_{2}-n_{1}-1}, b_{n_{1}}, \overline{1}^{n_{1}-1}, \ldots\right] .
$$


At this stage, we have constructed the first $2 n_{2}-1$ partial quotients of $\alpha$. Let $n_{3}>2 n_{2}$ be such that $\Psi\left((3 / 2)^{n}\right) \leq\left(10 b_{n_{2}}\right)^{-1}$ for any $n \geq n_{3}-1$. Then, set $b_{2 n_{2}}=\ldots=b_{n_{3}-1}=1$ and $b_{n_{3}}=\left\lceil 1 / \Psi\left(q_{n_{3}-1}\right)\right\rceil$. Observe that $b_{n_{3}} \geq 10 b_{n_{2}}$. Then, we again complete by symmetry, and we repeat our process in order to define $n_{4}, b_{n_{4}}$, and so on.

Clearly, the real number constructed in this way begins with infinitely many palindromes, thus it is either quadratic or transcendental by Theorem 1. Moreover, the assumption on the function $\varphi$ implies that $\alpha$ has unbounded partial quotients. It thus follows that it is transcendental. It remains for us to prove that it has the required property of approximation.

By (4.1), for any $j \geq 1$, we have

$$
\frac{\varphi\left(q_{n_{j}-1}\right)}{1+3 q_{n_{j}-1}^{2} \varphi\left(q_{n_{j}-1}\right)}<\left|\alpha-\frac{p_{n_{j}-1}}{q_{n_{j}-1}}\right|<\varphi\left(q_{n_{j}-1}\right) .
$$

Let $p_{n} / q_{n}$ with $n \geq n_{2}$ be a convergent to $\alpha$ not in the subsequence $\left(p_{n_{j}-1} / q_{n_{j}-1}\right)_{j \geq 1}$, and let $k$ be the integer defined by $n_{k}-1<n<n_{k+1}-1$. Then, by (4.1) and the way we defined the $b_{n_{j}}$, we have

$$
\begin{aligned}
\left|\alpha-\frac{p_{n}}{q_{n}}\right| & >\frac{1}{q_{n}^{2}\left(b_{n+1}+2\right)} \geq \frac{1}{q_{n}^{2}\left(b_{n_{k-1}}+2\right)} \\
& \geq \frac{1}{3 q_{n}^{2} b_{n_{k-1}}} \geq \frac{\varphi\left(q_{n}\right)}{3 q_{n_{k}-1}^{2} \varphi\left(q_{n_{k}-1}\right) b_{n_{k-1}}}
\end{aligned}
$$

since $x \mapsto x^{2} \varphi(x)$ is non-increasing. We then infer from (4.3) and

$$
b_{n_{k}-1} \leq \frac{b_{n_{k}}}{10} \leq \frac{11}{100} \cdot \frac{1}{q_{n_{k}-1}^{2} \varphi\left(q_{n_{k}-1}\right)}
$$

that

$$
\left|\alpha-\frac{p_{n}}{q_{n}}\right| \geq 3 \varphi\left(q_{n}\right) .
$$

To conclude, we observe that it follows from (4.2) that $\alpha$ is approximable at order $\varphi$, and from $(4.2),(4.4)$ and the fact that $\varphi$ is non-increasing that $\alpha$ is not approximable at any order $c \varphi$ with $0<c<1$. The proof of Theorem 5 is complete.

\section{Auxiliary results}

The proofs of Theorems 2 to 4 depend on a deep result from Diophantine approximation, namely the powerful Schmidt Subspace Theorem, stated as Theorem B below. However, we do not need the full force of this theorem to prove our Theorem 1: the transcendence criterion given by Theorem A is sufficient for our purpose. 
Theorem A. (W. M. Schmidt). Let $\alpha$ be a real number, which is neither rational, nor quadratic. If there exist a real number $w>3 / 2$ and infinitely many triples of integers $(p, q, r)$ with $q>0$ such that

$$
\max \left\{\left|\alpha-\frac{p}{q}\right|,\left|\alpha^{2}-\frac{r}{q}\right|\right\}<\frac{1}{q^{w}},
$$

then $\alpha$ is transcendental.

Proof : See [15].

Theorem B. (W. M. Schmidt). Let $m \geq 2$ be an integer. Let $L_{1}, \ldots, L_{m}$ be linearly independent linear forms in $\mathbf{x}=\left(x_{1}, \ldots, x_{m}\right)$ with algebraic coefficients. Let $\varepsilon$ be a positive real number. Then, the set of solutions $\mathbf{x}=\left(x_{1}, \ldots, x_{m}\right)$ in $\mathbf{Z}^{m}$ to the inequality

$$
\left|L_{1}(\mathbf{x}) \ldots L_{m}(\mathbf{x})\right| \leq\left(\max \left\{\left|x_{1}\right|, \ldots,\left|x_{m}\right|\right\}\right)^{-\varepsilon}
$$

lies in finitely many proper subspaces of $\mathbf{Q}^{m}$.

Proof : See e.g. [16] or [17].

For the reader convenience, we further recall some well-known results from the theory of continued fractions, whose proofs can be found e.g. in the book of Perron [13]. The seemingly innocent Lemma 1 appears to be crucial in the proofs of Theorems 2 to 4 .

Lemma 1. Let $\alpha=\left[0 ; a_{1}, a_{2}, \ldots\right]$ be a real number with convergents $\left(p_{\ell} / q_{\ell}\right)_{\ell \geq 1}$. Then, for any $\ell \geq 2$, we have

$$
\frac{q_{\ell-1}}{q_{\ell}}=\left[0 ; a_{\ell}, a_{\ell-1}, \ldots, a_{1}\right] .
$$

Lemma 2. Let $\alpha=\left[0 ; a_{1}, a_{2}, \ldots\right]$ and $\beta=\left[0 ; b_{1}, b_{2}, \ldots\right]$ be real numbers. Let $n \geq 1$ such that $a_{i}=b_{i}$ for any $i=1, \ldots, n$. We then have $|\alpha-\beta| \leq q_{n}^{-2}$, where $q_{n}$ denotes the denominator of the $n$-th convergent to $\alpha$.

For positive integers $a_{1}, \ldots, a_{m}$, we denote by $K_{m}\left(a_{1}, \ldots, a_{m}\right)$ the denominator of the rational number $\left[0 ; a_{1}, \ldots, a_{m}\right]$. It is commonly called a continuant.

Lemma 3. For any positive integers $a_{1}, \ldots, a_{m}$ and any integer $k$ with $1 \leq k \leq m-1$, we have

$$
K_{m}\left(a_{1}, \ldots, a_{m}\right)=K_{m}\left(a_{m}, \ldots, a_{1}\right)
$$

and

$$
\begin{aligned}
K_{k}\left(a_{1}, \ldots, a_{k}\right) \cdot K_{m-k}\left(a_{k+1}, \ldots, a_{m}\right) & \leq K_{m}\left(a_{1}, \ldots, a_{m}\right) \\
& \leq 2 K_{k}\left(a_{1}, \ldots, a_{k}\right) \cdot K_{m-k}\left(a_{k+1}, \ldots, a_{m}\right) .
\end{aligned}
$$

The following three results are needed for the proof of Theorem 4 . 
Lemma 4. Let $\alpha:=\left[0 ; a_{1}, a_{2}, \ldots, a_{n}\right]=p_{n} / q_{n}$ be a rational number $\alpha$. Then, we have

$$
\log q_{n}=\sum_{m=1}^{n} \log \left(\left[a_{m} ; a_{m-1}, \ldots, a_{1}\right]\right)
$$

Proof. It follows from Lemma 1 that

$$
\log q_{n}=\sum_{m=1}^{n} \log \left(\frac{q_{m}}{q_{m-1}}\right)=\sum_{m=1}^{n} \log \left(\left[a_{m} ; a_{m-1}, \ldots, a_{1}\right]\right),
$$

where $q_{0}, q_{1}, \ldots, q_{n}$ denote the denominators of the convergents of $\alpha$.

Throughout the rest of this Section, $\theta$ denotes the Golden Ratio $(1+\sqrt{5}) / 2$.

Lemma 5. Let $\alpha$ be a real number and denote by $\left(p_{n} / q_{n}\right)_{n \geq 1}$ the sequence of its convergents. Then, for every positive integer $k$, we have

$$
q_{k} \geq \frac{\theta^{k+1}}{2 \sqrt{5}}
$$

Proof. By definition of the convergents we have $q_{1}=1, q_{2}=a_{0}+1$ and $q_{n}=a_{n} q_{n-1}+q_{n-2}$, where $\left(a_{n}\right)_{n \geq 0}$ denotes the sequence of partial quotients of $\alpha$. We thus have $q_{1} \geq 1, q_{2} \geq 1$ and $q_{k} \geq q_{k-1}+q_{k-2}$ for every integer $k \geq 2$. For any integer $k>0$, set $s_{k}:=\theta^{k+1} /(2 \sqrt{5})$. Since $s_{1}<s_{2}<1$ and $s_{k+2}=s_{k+1}+s_{k}$, we get immediately that $q_{k} \geq s_{k}$ for any positive integer $k$.

Lemma 6. Let $r$ be a positive integer. Let $\left(x^{(k)}\right)_{1 \leq k \leq r}$ and $\left(y^{(k)}\right)_{1 \leq k \leq r}$ be two finite sequences of real numbers lying in $[1,+\infty)$. Denote by $\left[x_{1, k} ; x_{2, k}, \ldots\right]\left(\operatorname{resp} .\left[y_{1, k} ; y_{2, k}, \ldots\right]\right)$ the continued fraction expansion of $x^{(k)}$ (resp. of $\left.y^{(k)}\right)$. If, for every $k$ satisfying $1 \leq k \leq r$, we have

$$
x_{j, k}=y_{j, k}, \quad \text { for } j=1, \ldots, k,
$$

then,

$$
\sum_{k=1}^{r}\left|\log \left(x^{(k)}\right)-\log \left(y^{(k)}\right)\right|<\frac{20}{\theta^{3}}
$$

Proof. For every $k$ with $1 \leq k \leq r$, denote by $q_{k}$ the denominator of the $k$-th convergent of $x^{(k)}$. By assumption, $q_{k}$ is also the denominator of the $k$-th convergent of $y^{(k)}$. It thus follows from Lemma 2 that

$$
\left|x^{(k)}-y^{(k)}\right|<\frac{1}{q_{k}^{2}},
$$

which implies that

$$
1-\frac{1}{q_{k}^{2}} \leq \frac{x^{(k)}}{y^{(k)}} \leq 1+\frac{1}{q_{k}^{2}}
$$


We obtain

$$
\left|\log \left(x^{(k)}\right)-\log \left(y^{(k)}\right)\right|<\frac{1}{q_{k}^{2}}
$$

and it follows from Lemma 5

$$
\left|\log \left(x^{(k)}\right)-\log \left(y^{(k)}\right)\right|<\frac{20}{\theta^{2 k+2}}
$$

We thus get that

$$
\sum_{k=1}^{r}\left|\log \left(x^{(k)}\right)-\log \left(y^{(k)}\right)\right|<\frac{20}{\theta^{2}} \sum_{k=1}^{+\infty} \frac{1}{\theta^{2 k}}=\frac{20}{\theta^{3}},
$$

concluding the proof.

\section{Proof of Theorem 1}

Let $n$ be a positive integer. Denote by $p_{n} / q_{n}$ the $n$-th convergent to $\alpha$, that is, $p_{n} / q_{n}=\left[0 ; a_{1}, a_{2}, \ldots, a_{n}\right]$. By the theory of continued fraction, we have

$$
M_{n}:=\left(\begin{array}{cc}
q_{n} & q_{n-1} \\
p_{n} & p_{n-1}
\end{array}\right)=\left(\begin{array}{cc}
a_{1} & 1 \\
1 & 0
\end{array}\right)\left(\begin{array}{cc}
a_{2} & 1 \\
1 & 0
\end{array}\right) \ldots\left(\begin{array}{cc}
a_{n} & 1 \\
1 & 0
\end{array}\right)
$$

Since such a decomposition is unique, the matrix $M_{n}$ is symmetrical if, and only if, the word $a_{1} a_{2} \ldots a_{n}$ is a palindrome. Assume that this is case. Then, we have $p_{n}=q_{n-1}$. Recalling that

$$
\left|\alpha-\frac{p_{n}}{q_{n}}\right|<\frac{1}{q_{n}^{2}} \text { and } \quad\left|\alpha-\frac{p_{n-1}}{q_{n-1}}\right|<\frac{1}{q_{n-1}^{2}}
$$

we infer from $0<\alpha<1, a_{1}=a_{n},\left|p_{n} q_{n-1}-p_{n-1} q_{n}\right|=1$ and $q_{n} \leq\left(a_{n}+1\right) q_{n-1}$ that

$$
\begin{aligned}
\left|\alpha^{2}-\frac{p_{n-1}}{q_{n}}\right| & \leq\left|\alpha^{2}-\frac{p_{n-1}}{q_{n-1}} \cdot \frac{p_{n}}{q_{n}}\right| \leq\left|\alpha+\frac{p_{n-1}}{q_{n-1}}\right| \cdot\left|\alpha-\frac{p_{n}}{q_{n}}\right|+\frac{1}{q_{n} q_{n-1}} \\
& \leq 2\left|\alpha-\frac{p_{n}}{q_{n}}\right|+\frac{1}{q_{n} q_{n-1}}<\frac{a_{1}+3}{q_{n}^{2}},
\end{aligned}
$$

whence

$$
\max \left\{\left|\alpha-\frac{p_{n}}{q_{n}}\right|,\left|\alpha^{2}-\frac{p_{n-1}}{q_{n}}\right|\right\}<\frac{a_{1}+3}{q_{n}^{2}} .
$$

Consequently, if the sequence of the partial quotients of $\alpha$ begins in arbitrarily long palindromes, then (6.1) is satisfied for infinitely many integer triples $\left(p_{n}, q_{n}, p_{n-1}\right)$. By Theorem A, this shows that $\alpha$ is either quadratic or transcendental. 


\section{Proofs of Theorems 2 and 3}

Throughout the proofs of Theorems 2 and 3 , for any finite word $U=u_{1} \ldots u_{n}$ on $\mathbf{Z}_{\geq 1}$, we denote by $[0 ; U]$ the rational number $\left[0 ; u_{1}, \ldots, u_{n}\right]$.

Proof of Theorem 2. Keep the notation and the hypothesis of this theorem. Assume that the parameter $w>1$ is fixed, as well as the sequences $\left(U_{n}\right)_{n \geq 1}$ and $\left(V_{n}\right)_{n \geq 1}$ occurring in the definition of Condition $(*)_{w}$. Set also $r_{n}=\left|U_{n}\right|$ and $s_{n}=\left|U_{n} V_{n} \bar{U}_{n}\right|$, for any $n \geq 1$. We want to prove that the real number

$$
\alpha:=\left[0 ; a_{1}, a_{2}, \ldots\right]
$$

is transcendental. By assumption, we already know that $\alpha$ is irrational and not quadratic. Therefore, we assume that $\alpha$ is algebraic of degree at least three and we aim at deriving a contradiction.

Let $\left(p_{\ell} / q_{\ell}\right)_{\ell \geq 1}$ denote the sequence of convergents to $\alpha$. The key fact for the proof of Theorem 2 is the equality

$$
\frac{q_{\ell-1}}{q_{\ell}}=\left[0 ; a_{\ell}, a_{\ell-1}, \ldots, a_{1}\right],
$$

given by Lemma 1 . In other words, if $W_{\ell}$ denotes the prefix of length $\ell$ of the sequence a, then $q_{\ell-1} / q_{\ell}=\left[0 ; \overline{W_{\ell}}\right]$. Since, by assumption, we have

$$
\frac{p_{s_{n}}}{q_{s_{n}}}=\left[0 ; U_{n} V_{n} \overline{U_{n}}\right],
$$

we get that

$$
\frac{q_{s_{n}-1}}{q_{s_{n}}}=\left[0 ; U_{n} \overline{V_{n}} \overline{U_{n}}\right],
$$

and it follows from Lemma 2 that

$$
\left|q_{s_{n}} \alpha-q_{s_{n}-1}\right|<q_{s_{n}} q_{r_{n}}^{-2} .
$$

This shows in particular that

$$
\lim _{n \rightarrow+\infty} \frac{q_{s_{n}-1}}{q_{s_{n}}}=\alpha
$$

Furthermore, we clearly have

$$
\left|q_{s_{n}} \alpha-p_{s_{n}}\right|<q_{s_{n}}^{-1} \quad \text { and } \quad\left|q_{s_{n}-1} \alpha-p_{s_{n}-1}\right|<q_{s_{n}-1}^{-1} .
$$

Consider now the four linearly independent linear forms with algebraic coefficients:

$$
\begin{aligned}
& L_{1}\left(X_{1}, X_{2}, X_{3}, X_{4}\right)=\alpha X_{1}-X_{3}, \\
& L_{2}\left(X_{1}, X_{2}, X_{3}, X_{4}\right)=\alpha X_{2}-X_{4}, \\
& L_{3}\left(X_{1}, X_{2}, X_{3}, X_{4}\right)=\alpha X_{1}-X_{2}, \\
& L_{4}\left(X_{1}, X_{2}, X_{3}, X_{4}\right)=X_{2} .
\end{aligned}
$$


Evaluating them on the quadruple $\left(q_{s_{n}}, q_{s_{n}-1}, p_{s_{n}}, p_{s_{n}-1}\right)$, it follows from (7.1) and (7.3) that

$$
\prod_{1 \leq j \leq 4}\left|L_{j}\left(q_{s_{n}}, q_{s_{n}-1}, p_{s_{n}}, p_{s_{n}-1}\right)\right|<q_{r_{n}}^{-2} .
$$

By assumption, there exists a real number $M$ such that

$$
\sqrt{2} \leq q_{\ell}^{1 / \ell} \leq M
$$

for any integer $\ell \geq 3$. Thus, for any integer $n \geq 3$, we have

$$
q_{r_{n}} \geq \sqrt{2}^{r_{n}} \geq\left(M^{s_{n}}\right)^{\left(r_{n} \log \sqrt{2}\right) /\left(s_{n} \log M\right)} \geq q_{s_{n}}^{\left(r_{n} \log \sqrt{2}\right) /\left(s_{n} \log M\right)}
$$

and we infer from (7.4) and from $(i i)$ of Condition $(*)_{w}$ that

$$
\prod_{1 \leq j \leq 4}\left|L_{j}\left(q_{s_{n}}, q_{s_{n}-1}, p_{s_{n}}, p_{s_{n}-1}\right)\right| \ll q_{s_{n}}^{-\varepsilon}
$$

holds for some positive real number $\varepsilon$.

It then follows from Theorem B that the points $\left(q_{s_{n}}, q_{s_{n}-1}, p_{s_{n}}, p_{s_{n}-1}\right)$ lie in a finite number of proper subspaces of $\mathbf{Q}^{4}$. Thus, there exist a non-zero integer quadruple $\left(x_{1}, x_{2}, x_{3}, x_{4}\right)$ and an infinite set of distinct positive integers $\mathcal{N}_{1}$ such that

$$
x_{1} q_{s_{n}}+x_{2} q_{s_{n}-1}+x_{3} p_{s_{n}}+x_{4} p_{s_{n}-1}=0,
$$

for any $n$ in $\mathcal{N}_{1}$. Dividing (7.5) by $q_{s_{n}}$, we obtain

$$
x_{1}+x_{2} \frac{q_{s_{n}-1}}{q_{s_{n}}}+x_{3} \frac{p_{s_{n}}}{q_{s_{n}}}+x_{4} \frac{p_{s_{n}-1}}{q_{s_{n}-1}} \cdot \frac{q_{s_{n}-1}}{q_{s_{n}}}=0 .
$$

By letting $n$ tend to infinity along $\mathcal{N}_{1}$ in (7.6), it follows from (7.2) that

$$
x_{1}+\left(x_{2}+x_{3}\right) \alpha+x_{4} \alpha^{2}=0 .
$$

Since, by assumption, $\alpha$ is not a quadratic number, we have $x_{1}=x_{4}=0$ and $x_{2}=-x_{3}$. Then, (7.5) implies that

$$
q_{s_{n}-1}=p_{s_{n}} .
$$

Consider now the three linearly independent linear forms with algebraic coefficients:

$$
L_{1}^{\prime}\left(Y_{1}, Y_{2}, Y_{3}\right)=\alpha Y_{1}-Y_{2}, \quad L_{2}^{\prime}\left(Y_{1}, Y_{2}, Y_{3}\right)=\alpha Y_{2}-Y_{3}, \quad L_{3}^{\prime}\left(Y_{1}, Y_{2}, Y_{3}\right)=Y_{1} .
$$

Evaluating them on the triple $\left(q_{s_{n}}, p_{s_{n}}, p_{s_{n}-1}\right)$, we infer from (7.3) and (7.7) that

$$
\prod_{1 \leq j \leq 3}\left|L_{j}^{\prime}\left(q_{s_{n}}, p_{s_{n}}, p_{s_{n}-1}\right)\right|<q_{s_{n}-1}^{-1} \ll q_{s_{n}}^{-0.9},
$$


since we have

$$
q_{\ell+1} \ll q_{\ell}^{1.1}, \quad \text { for any } \ell \geq 1,
$$

by Roth's Theorem. Here, the constants implied by $\ll$ depend only on $\alpha$.

It then follows from Theorem B that the points $\left(q_{s_{n}}, p_{s_{n}}, p_{s_{n}-1}\right)$ with $n$ in $\mathcal{N}_{1}$ lie in a finite number of proper subspaces of $\mathbf{Q}^{3}$. Thus, there exist a non-zero integer triple $\left(y_{1}, y_{2}, y_{3}\right)$ and an infinite set of distinct positive integers $\mathcal{N}_{2}$ such that

$$
y_{1} q_{s_{n}}+y_{2} p_{s_{n}}+y_{3} p_{s_{n}-1}=0,
$$

for any $n$ in $\mathcal{N}_{2}$. Dividing (7.8) by $q_{s_{n}}$, we get

$$
y_{1}+y_{2} \frac{p_{s_{n}}}{q_{s_{n}}}+y_{3} \frac{p_{s_{n}-1}}{q_{s_{n}-1}} \cdot \frac{q_{s_{n}-1}}{q_{s_{n}}}=0 .
$$

By letting $n$ tend to infinity along $\mathcal{N}_{2}$, it thus follows from (7.7) and (7.9) that

$$
y_{1}+y_{2} \alpha+y_{3} \alpha^{2}=0 \text {. }
$$

Since $\left(y_{1}, y_{2}, y_{3}\right)$ is a non-zero triple of integers, we have reached a contradiction. Consequently, the real number $\alpha$ is transcendental. This completes the proof of the theorem.

Proof of Theorem 3. Keep the notation and the hypothesis of this theorem. Assume that the parameters $w$ and $w^{\prime}$ are fixed, as well as the sequences $\left(U_{n}\right)_{n \geq 1},\left(V_{n}\right)_{n \geq 1}$ and $\left(W_{n}\right)_{n \geq 1}$. Set also $r_{n}=\left|W_{n}\right|, s_{n}=\left|W_{n} U_{n}\right|$ and $t_{n}=\left|W_{n} U_{n} V_{n} \overline{U_{n}}\right|$, for any $n \geq 1$. We want to prove that the real number

$$
\alpha:=\left[0 ; a_{0}, a_{1}, a_{2}, \ldots\right]
$$

is transcendental. By assumption, we already know that $\alpha$ is irrational and not quadratic. Therefore, we assume that $\alpha$ is algebraic of degree at least three and we aim at deriving a contradiction. Throughout this Section, the constants implied by $\ll$ depend only on $\alpha$. In view of Theorem 2 , we may assume that $r_{n} \geq 1$ for any $n$.

The key idea for our proof is to consider, for any positive integer $n$, the rational $P_{n} / Q_{n}$ defined by

$$
\frac{P_{n}}{Q_{n}}:=\left[0 ; W_{n} U_{n} V_{n} \overline{U_{n}} \overline{W_{n}}\right]
$$

and to use the fact that the word $W_{n} U_{n} V_{n} \overline{U_{n}} \overline{W_{n}}$ is a quasi-palindrome. Let $P_{n}^{\prime} / Q_{n}^{\prime}$ denote the last convergent to $P_{n} / Q_{n}$. By assumption we have

$$
\frac{p_{t_{n}}}{q_{t_{n}}}=\left[0 ; W_{n} U_{n} V_{n} \overline{U_{n}}\right]
$$

and it thus follows from Lemma 2 that

$$
\left|Q_{n} \alpha-P_{n}\right|<Q_{n} q_{t_{n}}^{-2}
$$


and

$$
\left|Q_{n}^{\prime} \alpha-P_{n}^{\prime}\right|<Q_{n}^{\prime} q_{t_{n}}^{-2},
$$

since $\bar{W}_{n}$ has at least one letter. Furthermore, Lemma 1 implies that

$$
\frac{Q_{n}^{\prime}}{Q_{n}}=\left[0 ; W_{n} U_{n} \overline{V_{n}} \overline{U_{n}} \overline{W_{n}}\right],
$$

and we get from Lemma 2 that

$$
\left|Q_{n} \alpha-Q_{n}^{\prime}\right|<Q_{n} q_{s_{n}}^{-2}
$$

This shows in particular that

$$
\lim _{n \rightarrow+\infty} \frac{Q_{n}^{\prime}}{Q_{n}}=\alpha .
$$

Consider now the following four linearly independent linear forms with algebraic coefficients:

$$
\begin{aligned}
& L_{1}\left(X_{1}, X_{2}, X_{3}, X_{4}\right)=\alpha X_{1}-X_{3}, \\
& L_{2}\left(X_{1}, X_{2}, X_{3}, X_{4}\right)=\alpha X_{2}-X_{4}, \\
& L_{3}\left(X_{1}, X_{2}, X_{3}, X_{4}\right)=\alpha X_{1}-X_{2}, \\
& L_{4}\left(X_{1}, X_{2}, X_{3}, X_{4}\right)=X_{2} .
\end{aligned}
$$

Evaluating them on the quadruple $\left(Q_{n}, Q_{n}^{\prime}, P_{n}, P_{n}^{\prime}\right)$, it follows from (7.10), (7.11) and (7.12) that

$$
\prod_{1 \leq j \leq 4}\left|L_{j}\left(Q_{n}, Q_{n}^{\prime}, P_{n}, P_{n}^{\prime}\right)\right|<Q_{n}^{4} q_{t_{n}}^{-4} q_{s_{n}}^{-2} .
$$

We infer from Lemma 3 that

$$
q_{t_{n}} q_{r_{n}} \leq Q_{n} \leq 2 q_{t_{n}} q_{r_{n}} \quad \text { and } \quad q_{s_{n}}^{2} \leq Q_{n} \leq q_{t_{n}}^{2},
$$

and thus (7.14) gives

$$
\prod_{1 \leq j \leq 4}\left|L_{j}\left(Q_{n}, Q_{n}^{\prime}, P_{n}, P_{n}^{\prime}\right)\right| \ll q_{r_{n}}^{4} q_{s_{n}}^{-2} .
$$

Moreover, by our assumption (2.1), there exists $\eta>0$ such that, for any $n$ large enough, we have

$$
\left|U_{n}\right| \geq\left(2 \frac{\log M}{\log m} \cdot \frac{1+\eta}{1-\eta}-1\right)\left|W_{n}\right| .
$$

Consequently, assuming that $n$ is sufficiently large, we get

$$
m^{(1-\eta) s_{n}} \geq M^{2(1+\eta) r_{n}}
$$

and

$$
q_{s_{n}} \geq q_{r_{n}}^{2+\eta^{\prime}},
$$


for some positive real number $\eta^{\prime}$. It then follows from (7.16) that

$$
\prod_{1 \leq j \leq 4}\left|L_{j}\left(Q_{n}, Q_{n}^{\prime}, P_{n}, P_{n}^{\prime}\right)\right| \ll q_{s_{n}}^{-2 \eta^{\prime} /\left(2+\eta^{\prime}\right)}
$$

By assumption, we have for any $\ell$ large enough

$$
\sqrt{2} \leq q_{\ell}^{1 / \ell} \leq 2 M
$$

Thus, for any integer $n$ large enough, we have

$$
\begin{aligned}
q_{s_{n}} \geq \sqrt{2}^{s_{n}} \geq\left((2 M)^{t_{n}}\right)^{\left(s_{n} \log \sqrt{2}\right) /\left(t_{n} \log 2 M\right)} & \geq q_{t_{n}}^{\left(s_{n} \log \sqrt{2}\right) /\left(t_{n} \log 2 M\right)} \\
& \geq Q_{n}^{\left(s_{n} \log \sqrt{2}\right) /\left(2 t_{n} \log 2 M\right)}
\end{aligned}
$$

by (7.15). We then infer from (7.17) and from (ii) of Condition $(*)_{w, w^{\prime}}$ that

$$
\prod_{1 \leq j \leq 4}\left|L_{j}\left(Q_{n}, Q_{n}^{\prime}, P_{n}, P_{n}^{\prime}\right)\right| \ll Q_{n}^{-\varepsilon}
$$

holds for some positive $\varepsilon$.

It then follows from Theorem B that the points $\left(Q_{n}, Q_{n}^{\prime}, P_{n}, P_{n}^{\prime}\right)$ lie in a finite number of proper subspaces of $\mathbf{Q}^{4}$. Thus, there exist a non-zero integer quadruple $\left(x_{1}, x_{2}, x_{3}, x_{4}\right)$ and an infinite set of distinct positive integers $\mathcal{N}_{1}$ such that

$$
x_{1} Q_{n}+x_{2} Q_{n}^{\prime}+x_{3} P_{n}+x_{4} P_{n}^{\prime}=0,
$$

for any $n$ in $\mathcal{N}_{1}$. Dividing by $Q_{n}$, we obtain

$$
x_{1}+x_{2} \frac{Q_{n}^{\prime}}{Q_{n}}+x_{3} \frac{P_{n}}{Q_{n}}+x_{4} \frac{P_{n}^{\prime}}{Q_{n}^{\prime}} \cdot \frac{Q_{n}^{\prime}}{Q_{n}}=0 .
$$

By letting $n$ tend to infinity along $\mathcal{N}_{1}$, we infer from (7.13) that

$$
x_{1}+\left(x_{2}+x_{3}\right) \alpha+x_{4} \alpha^{2}=0 .
$$

Since $\left(x_{1}, x_{2}, x_{3}, x_{4}\right) \neq(0,0,0,0)$ and since $\alpha$ is irrational and not quadratic, we have $x_{1}=x_{4}=0$ and $x_{2}=-x_{3}$. Then, (7.19) implies that

$$
Q_{n}^{\prime}=P_{n} .
$$

Consider now the following three linearly independent linear forms with algebraic coefficients:

$$
L_{1}^{\prime}\left(Y_{1}, Y_{2}, Y_{3}\right)=\alpha Y_{1}-Y_{2}, L_{2}^{\prime}\left(Y_{1}, Y_{2}, Y_{3}\right)=\alpha Y_{2}-Y_{3}, L_{3}^{\prime}\left(Y_{1}, Y_{2}, Y_{3}\right)=Y_{1} .
$$


Evaluating them on the quadruple $\left(Q_{n}, P_{n}, P_{n}^{\prime}\right)$, it follows from (7.10), (7.11), (7.15) and (7.20) that

$$
\prod_{1 \leq j \leq 3}\left|L_{j}\left(Q_{n}, P_{n}, P_{n}^{\prime}\right)\right| \ll Q_{n}^{3} q_{t_{n}}^{-4} \ll q_{r_{n}}^{4} Q_{n}^{-1} \ll q_{r_{n}}^{4} q_{s_{n}}^{-2} \ll Q_{n}^{-\varepsilon},
$$

with the same $\varepsilon$ as in (7.18). It then follows from Theorem B that the points $\left(Q_{n}, P_{n}, P_{n}^{\prime}\right)$ lie in a finite number of proper subspaces of $\mathbf{Q}^{3}$. Thus, there exist a non-zero integer triple $\left(y_{1}, y_{2}, y_{3}\right)$ and an infinite set of distinct positive integers $\mathcal{N}_{2}$ such that

$$
y_{1} Q_{n}+y_{2} P_{n}+y_{3} P_{n}^{\prime}=0
$$

for any $n$ in $\mathcal{N}_{2}$. We then proceed exactly as at the end of the proof of Theorem 2 to reach a contradiction. This finishes the proof of our theorem.

\section{Proof of Theorem 4}

This Section is devoted to the proof of Theorem 4. Instead of a straightforward application of Theorem 3, which would yield a weaker result, we carefully determine here the growth of the denominators of the convergents to the real numbers under consideration.

Proof of Theorem 4. Let $\alpha=\alpha_{\boldsymbol{\Lambda}}=\left[0 ; \bar{a}^{\lambda_{1}}, \bar{b}^{\lambda_{2}}, \bar{a}^{\lambda_{3}}, \ldots, \bar{a}^{\lambda_{2 n-1}}, \bar{b}^{\lambda_{2 n}}, \ldots\right]$ and denote by $\left(p_{\ell} / q_{\ell}\right)_{\ell \geq 1}$ the sequence of its convergents. Assume that

$$
\liminf _{n \rightarrow \infty} \frac{\lambda_{n+1}}{\lambda_{n}}>\sqrt{2}
$$

We first remark that if moreover the sequence $\left(\lambda_{n+1} / \lambda_{n}\right)_{n \geq 1}$ is not bounded from above, then the transcendence of $\alpha$ follows from a direct application of Theorem 3. Indeed, let us assume that there exists an increasing sequence of positive integers $\left(n_{k}\right)_{k \geq 1}$ such that $\lim _{k \rightarrow \infty}\left(\lambda_{n_{k}+1} / \lambda_{n_{k}}\right)=+\infty$. Without loss of generality, we can also assume that $n_{k}$ is odd for every $k \geq 1$. Then, we apply Theorem 3 with $W_{k}=\bar{a}^{\lambda_{1}} \bar{b}^{\lambda_{2}} \bar{a}^{\lambda_{3}} \ldots \bar{a}^{\lambda_{n_{k}}}, U_{k}=\bar{b}^{\left\lfloor\lambda_{n_{k}+1}\right\rfloor / 2}$ and $V_{k}$ equals to the empty word, since $\left|V_{k}\right| /\left|U_{k}\right|=0$ and $\lim _{k \rightarrow \infty}\left|U_{k}\right| /\left|W_{k}\right|=+\infty$.

From now on, we thus assume that

$$
\limsup _{n \rightarrow \infty} \frac{\lambda_{n+1}}{\lambda_{n}}<+\infty
$$

Without loss of generality, we can assume that $b>a$. For $n \geq 1$, we set $W_{n}=$ $\bar{a}^{\lambda_{1}} \bar{b}^{\lambda_{2}} \bar{a}^{\lambda_{3}} \ldots \bar{b}^{\lambda_{2 n-2}}, U_{n}=\bar{a}^{\lambda_{2 n-1}} \bar{b}^{\lambda_{2 n}}$ and $V_{n}=\bar{a}^{\lambda_{2 n+1}} \bar{b}^{\lambda_{2 n+2}-\lambda_{2 n}}$. Set also $r_{n}=\left|W_{n}\right|$, $s_{n}=\left|W_{n} U_{n}\right|$ and $t_{n}=\left|W_{n} U_{n} V_{n} \bar{U}_{n}\right|$. By assumption, we have

$$
p_{t_{n}} / q_{t_{n}}=\left[0 ; W_{n} U_{n} V_{n} \bar{U}_{n}\right] .
$$


Following the idea introduced in the proof of Theorem 3, we consider the rational $P_{n} / Q_{n}$ defined by

$$
P_{n} / Q_{n}=\left[0 ; W_{n} U_{n} V_{n} \bar{U}_{n} \overline{W_{n}}\right] .
$$

Then, using the fact that the word $W_{n} U_{n} V_{n} \bar{U}_{n} \overline{W_{n}}$ is a quasi-palindrome, we can mimic the first steps of the proof of Theorem 3 (see in particular (7.16)). We obtain that $\alpha$ is transcendental as soon as we can ensure the existence of a positive real number $\varepsilon$ such that

$$
q_{r_{n}}^{4} q_{s_{n}}^{-2} \ll Q_{n}^{-\varepsilon} .
$$

Here and below, the numerical constant implicit in $\ll$ does not depend on $n$. Moreover, we can deduce from (8.1) and (8.2) that the sequence $\left(\left|U_{n} V_{n}\right| /\left|W_{n}\right|\right)_{n \geq 1}$ is bounded from above which implies the existence of a positive real number $\eta$ such that

$$
q_{r_{n}} \ll Q_{n}^{\eta} .
$$

It thus follows from (8.3) that $\alpha$ is transcendental as soon as we can ensure the existence of a positive real number $\delta$ such that

$$
q_{r_{n}}^{4} q_{s_{n}}^{-2} \ll q_{r_{n}}^{-\delta} .
$$

Let $\left(a_{\ell}\right)_{\ell \geq 1}$ denote the sequence of partial quotients of $\alpha$, and, for every $m \geq 1$, set $x_{m}=\left[a_{m} ; a_{m-1}, \ldots, a_{1}\right]$. It follows from Lemma 4 that

$$
\log q_{s_{n}}=\sum_{m=1}^{s_{n}} \log x_{m}=\log q_{r_{n}}+\sum_{m=r_{n}+1}^{s_{n}} \log x_{m} .
$$

In view of (8.4), it only remains for us to prove that there exists a positive real number $\delta$ such that

$$
\sum_{m=r_{n}+1}^{s_{n}} \log x_{m} \geq(1+\delta) \sum_{m=1}^{r_{n}} \log x_{m}
$$

for $n$ large enough.

In the sequel of the proof, we show that (8.5) holds. For $n \geq 1$, set $c_{n}=\sum_{k=1}^{n} \lambda_{k}$, $d_{n}=\sum_{k=1}^{n} \lambda_{2 k}$ and $e_{n}=\sum_{k=1}^{n} \lambda_{2 k-1}$. To simplify the exposition, we put $c_{0}=d_{0}=e_{0}=0$ and, for $0 \leq j \leq n-2$, we set

$$
A_{2 j+1}=\sum_{m=c_{2 j}+1}^{c_{2 j+1}} \log x_{m}, \quad B_{2 j+2}=\sum_{m=c_{2 j+1}+1}^{c_{2 j+2}} \log x_{m},
$$

and, for $0 \leq j \leq n-2$, we set

$$
A_{2 j+1}^{\prime}=\sum_{m=r_{n}+e_{j}+1}^{r_{n}+e_{j+1}} \log x_{m}, \quad B_{2 j+2}^{\prime}=\sum_{m=r_{n}+\lambda_{2 n-1}+d_{j}+1}^{r_{n}+\lambda_{2 n-1}+d_{j+1}} \log x_{m} .
$$


It follows from (1) that for $0 \leq j \leq n-2$ and for $r_{n}+e_{j}+1 \leq m \leq r_{n}+e_{j+1}$, the first $m$ partial quotients of $x_{m}$ are all equals to $a$. We similary deduce from (1) that for $0 \leq j \leq n-2$ and for $r_{n}+\lambda_{2 n-1}+d_{j}+1 \leq m \leq r_{n}+\lambda_{2 n-1}+d_{j+1}$, the first $m$ partial quotients of $x_{m}$ are all equal to $b$. By Lemma 6 , we thus have

$$
\begin{aligned}
& \left|A_{2 j+1}-\log \alpha_{a}\right|<\frac{20}{\theta^{3}}, \quad\left|A_{2 j+1}^{\prime}-\log \alpha_{a}\right|<\frac{20}{\theta^{3}} \\
& \left|B_{2 j+2}-\log \alpha_{b}\right|<\frac{20}{\theta^{3}}, \quad\left|B_{2 j+2}^{\prime}-\log \alpha_{b}\right|<\frac{20}{\theta^{3}}
\end{aligned}
$$

where, as in Section 5 , we have set $\theta=(1+\sqrt{5}) / 2$. On the other hand, we have

$$
\sum_{m=1}^{r_{n}} \log x_{m}=\sum_{j=0}^{n-2}\left(A_{2 j+1}+B_{2 j+2}\right)
$$

and

$$
\sum_{m=r_{n}+1}^{s_{n}} \log x_{m}=\sum_{j=0}^{n-2}\left(A_{2 j+1}^{\prime}+B_{2 j+2}^{\prime}\right)+\sum_{m=r_{n}+e_{n-1}+1}^{r_{n}+\lambda_{2 n-1}} \log x_{m}+\sum_{m=s_{n}-\lambda_{2 n}+d_{n-1}+1}^{s_{n}} \log x_{m} .
$$

Then, (8.6) and (8.7) imply that

$$
\sum_{m=r_{n}+1}^{s_{n}} \log x_{m}-\sum_{m=1}^{r_{n}} \log x_{m} \geq \sum_{m=r_{n}+e_{n-1}+1}^{r_{n}+\lambda_{2 n-1}} \log x_{m}+\sum_{m=s_{n}-\lambda_{2 n}+d_{n-1}+1}^{s_{n}} \log x_{m}-\frac{80 n}{\theta^{3}}
$$

We also deduce from (8.1) the existence of a positive real number $\omega$ such that

$$
\lambda_{2 n-1}>(1+\omega) e_{n-1} \text { and } \quad \lambda_{2 n}>(1+\omega) d_{n-1} .
$$

Now, from $(8.8)$ and the fact that $x_{m} \geq(b+2) /(b+1)$ for any $m \geq 2$, it thus follows

$$
\sum_{m=r_{n}+1}^{s_{n}} \log x_{m}-\sum_{m=1}^{r_{n}} \log x_{m} \geq w\left(e_{n-1}+d_{n-1}\right) \log \frac{b+2}{b+1}-\frac{80 n}{\theta^{3}}=\omega r_{n} \log \frac{b+2}{b+1}-\frac{80 n}{\theta^{3}} .
$$

We easily deduce from (8.1) that $r_{n} \geq 2^{n}$ for $n$ large enough, which implies that

$$
\sum_{m=r_{n}+1}^{s_{n}} \log x_{m}-\sum_{m=1}^{r_{n}} \log x_{m} \geq \frac{\omega}{2} r_{n} \log \frac{b+2}{b+1}
$$

for $n$ large enough. On the other hand, we have $x_{m}<b+1$ for every positive integer $m$, implying that

$$
\sum_{m=1}^{r_{n}} \log x_{m}<r_{n} \log (b+1)
$$


Combining (8.9) and (8.10), we get the existence of a positive real number $\delta$ such that

$$
\sum_{m=r_{n}+1}^{s_{n}} \log x_{m}-\sum_{m=1}^{r_{n}} \log x_{m}>\delta\left(\sum_{m=1}^{r_{n}} \log x_{m}\right),
$$

for $n$ large enough. In view of (8.5), this concludes the proof.

\section{References}

[1] B. Adamczewski \& Y. Bugeaud, On the complexity of algebraic numbers. II. Continued fractions, Acta Math. To appear.

[2] B. Adamczewski \& Y. Bugeaud, A short proof of the transcendence of the ThueMorse continued fraction. Preprint.

[3] J.-P. Allouche, J. L. Davison, M. Queffélec \& L. Q. Zamboni, Transcendence of Sturmian or morphic continued fractions, J. Number Theory 91 (2001), 39-66.

[4] A. Baker, Continued fractions of transcendental numbers, Mathematika 9 (1962), $1-8$.

[5] A. Baker, On Mahler's classification of transcendental numbers, Acta Math. 111 (1964), 97-120.

[6] J. L. Davison, A class of transcendental numbers with bounded partial quotients. In R. A. Mollin, ed., Number Theory and Applications, pp. 365-371, Kluwer Academic Publishers, 1989.

[7] V. Jarník, Über die simultanen Diophantische Approximationen, Math. Z. 33 (1931), $505-543$.

[8] A. Ya. Khintchine, Continued fractions, Gosudarstv. Izdat. Tehn.-Theor. Lit. Moscow-Leningrad, 2nd edition, 1949 (in Russian).

[9] S. Lang, Introduction to Diophantine Approximations, Springer-Verlag (1995).

[10] W. J. LeVeque, Topics in Number Theory, Vols. 1 \& 2, Addison-Wesley Publ. Co., Inc., Reading, MA.

[11] J. Liouville, Sur des classes très étendues de quantités dont la valeur n'est ni algébrique, ni même réductible à des irrationelles algébriques, C. R. Acad. Sci. Paris 18 (1844), 883-885 and 993-995.

[12] E. Maillet, Introduction à la théorie des nombres transcendants et des propriétés arithmétiques des fonctions, Gauthier-Villars, Paris, 1906.

[13] O. Perron, Die Lehre von den Ketterbrüchen. Teubner, Leipzig, 1929. 
[14] M. Queffélec, Transcendance des fractions continues de Thue-Morse, J. Number Theory 73 (1998), 201-211.

[15] W. M. Schmidt, On simultaneous approximations of two algebraic numbers by rationals, Acta Math. 119 (1967), 27-50.

[16] W. M. Schmidt, Norm form equations, Ann. of Math. 96 (1972), 526-551.

[17] W. M. Schmidt, Diophantine approximation, Lecture Notes in Mathematics 785, Springer, Berlin, 1980.

Boris Adamczewski

CNRS, Institut Camille Jordan

Université Claude Bernard Lyon 1

Bât. Braconnier, 21 avenue Claude Bernard 69622 VILLEURANNE Cedex (FRANCE)

Boris.Adamczewski@math.univ-lyon1.fr
Yann Bugeaud Université Louis Pasteur U. F. R. de mathématiques 7, rue René Descartes 67084 STRASBOURG Cedex (FRANCE) bugeaud@math.u-strasbg.fr

Institut für Diskrete Mathematik und Geometrie TU Wien

Wiedner Hauptstrasse 8-10 1040 WIEN (AUSTRIA) bugeaud@geometrie.tuwien.ac .at 